\title{
PWM GENERATION TO CONTROL VARIABLE FREQUENCY POWER SOURCE
}

\author{
Ananya Devraj ${ }^{1}$, Darshan $\mathbf{R}^{2}$ \\ ${ }^{1}$ Mtech scholar, VLSI Design \& Embedded Systems, T John College, Karnataka, India \\ ${ }^{2}$ Mtech scholar, Power System Engineering, SDMCET, Karnataka, India
}

\begin{abstract}
The efficient use of available power for various types of demand has remained an important issue in electrical engineering domain. The focus has very often been to develop dedicated types of power sources according to specific, tailor-made demands. Very often, such requirements are specified in the form of generation of AC sources having variable magnitude, variable frequency and different types of waveform shapes. Depending on requirement, there may be design and development of single phase and lor three phase power sources.

Presently available power generation sources have no real time implementation. Moreover these are not cost effective. The quality of electrical power developed is gradually becoming a matter of major concern as the new generation equipment installed for increasing productivity very often turn out to be the major sources of creating additional power quality problems. Hence, in an overall consideration, design of high quality, variable voltage variable frequency power source with a wide range of peak-topeak alternating waveforms, with a wide range of frequency and with minimum distortion in the generated waveform, still remains a major challenge. The present work describes the development of a microcontroller-based variable voltage variable frequency power source, which is a necessity for various applications. The power source is developed using H-bridge inverter, which uses four MOSFETS for this purpose. The design utilizes the concept of generating a special kind of signals for the driver circuit. This special signal is nothing but a pulse width modulated signal which is provided as an input to the driver. The H-bridge inverter along with the microcontroller incorporates a memory that is based on a table. The memory used in this case is a Read Only Memory (ROM) that uses Look-Up-Table.
\end{abstract}

Keywords: H-bridge inverter, Microcontroller, Pulse Width Modulation, and Look-Up table.

\section{INTRODUCTION}

These days, electrical equipment has become an important part of daily life. Any electrical apparatus basically require power, without which it is unable to work. This urges the necessity or the importance of power source. Different types of equipment's require different types of power source. Thus for various applications versatile power source is required, which is the need of the day. This chapter deals with pulse width modulated signals and introduction for the handling microcontroller using these signals.

\subsection{Pulse Width Modulation (PWM) Technique}

PWM technique [2] is characterized by the generation of the constant amplitude pulses in which the pulse duration is modulated to obtain necessary specific waveform. This is the most efficient method of controlling the output signal by controlling the width of the pulses. Advantages of PWM technique [2]:

- When used in the inverters, the output voltage control can be obtained without any additional components.

- With this type of control, lower order harmonics can be eliminated or minimized along with its output voltage control.

\subsection{PWM using Microcontroller}

The most common and popular technique of digital puresine wave generation is pulse-width-modulation [2]. The Pulse Width Modulation technique involves generation of a digital waveform, for which the duty-cycle is modulated such that the average voltage of the waveform corresponds to a pure sine wave. This is a method of generating a signal that is $\operatorname{logic} 1$ for a portion of the time and logic 0 for the remainder of the time. Pulse Width Modulation is an important and essential concept in embedded systems. Microcontrollers can be used for applications like speed control of motors, brightness control in bulbs, LED's etc., this is done by providing a train of controlled pulses by programming the microcontroller [1]. Most of the PWM controllers available in the market generally have lower frequency range, starting from few hundreds of Hertz and hence are not fit to meet the requirements of the system. Such low frequencies can be precisely generated using the inbuilt timers provided in the microcontroller. Another important requirement of frequency driver is to generate dead time. A precise dead time can be easily generated through a microcontroller. 


\section{METHODOLOGY}

The proposed scheme uses a concept of generating suitable PWM [2] signals, called pulse width modulation (PWM) technique, where constant amplitude pulses are generated with different duty cycles for each period. The block diagram of the methodology employed is as shown in the Fig-2.1.

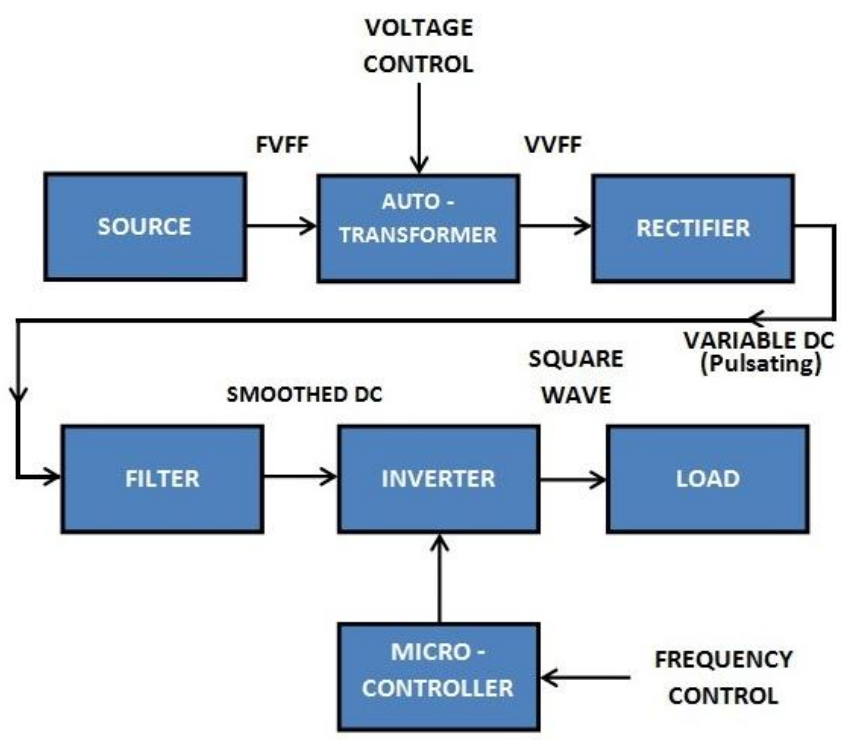

FVFF: Fixed Voltage Fixed Frequency VVFF: Variable Voltage Fixed Frequency

Fig -2.1: Block diagram of the variable frequency source

Source: The source is nothing but the supply from the mains, which is constant in terms of its frequency and almost constant in terms of its voltage. It can be otherwise said as fixed voltage fixed frequency source which provide a fixed rating of power to equipment. AC power supply usually uses a transformer to convert the voltage from the AC mains to a different, usually lower, voltage. These days, common electrical operations like speed control of motors, brightness control etc, may require continuous variation in source with respect to its frequency and voltage level or value. Source may be DC or AC. AC source has been considered in this work, since it is readily available for both household and industrial use. The source remains constant, for any load end variations. Usually in India the source available is of $230 \mathrm{~V}$, single phase, $50 \mathrm{~Hz}$ or $440 \mathrm{~V}$, three phase, $50 \mathrm{~Hz}$. Therefore, in general a $230 \mathrm{~V}$, single phase, $50 \mathrm{~Hz}$ is considered as the source for the present work.

Auto-transformer: The source, usually AC obtained from the mains is provided to this as input. The constant frequency, constant magnitude voltage input given to this equipment may be converted into required level of voltage. This voltage level may be higher than that of the input voltage or lower than it. This is done with the help of a knob present on top of the auto-transformer, by rotating it either clockwise (to increase the magnitude of the input) or anti-clockwise (to decrease the magnitude of the input). The frequency of the source remains constant throughout the process.
Rectifier: Rectification basically refers to the conversion of $\mathrm{AC}$ to pulsating DC and the device that does this function is called as a rectifier [4]. Usually rectifiers are constructed to obtain DC voltages from the $230 \mathrm{~V}$ AC mains. Thus a step down transformer invariably forms an integral part of any rectifier. The output of rectifier makes only unidirectional excursions. Rectifiers are especially useful in the design of DC power supplies required for all electronic circuits to work. It is an alternative to a battery wherein DC voltages can be obtained from the AC mains. A semiconductor diode can be used to convert an input AC waveform with zero average value into a unidirectional waveform with non-zero average value. It is of 2 type's half-wave rectifier and fullwave rectifier.

Half-wave rectifier [4]: The process of removing one half of the input signal, such as the entire waveform in one direction of a sinusoidal signal, to establish a DC level is called half-wave rectification. And the device used for this cause is called as half-wave rectifier. Half-wave rectifier usually consists of a diode, the diode conducts only during one half-cycle of the input AC signal and remains off in the next half-cycle of input AC signal.

Full-wave rectifier: Two half-wave rectifiers may be used to build a full-wave rectifier [4]. One of the two diodes works (on) during positive half cycle, while the other one stays off and similarly the second diode remains on during negative half cycle, while the first one stays off.

Filter: A first order RC filter is used to eliminate the AC component present in the rectified output, thereby providing a smoothed DC.

Inverter: Basically they are DC to AC converters, commonly called as Inverters. The inverter performs the opposite function of a rectifier. It is so named because early mechanical AC to DC converters were made to work in reverse, and thus were "inverted", to convert DC to AC. Usually the input to the inverters is fixed DC voltage. The output of the inverter is the fixed or variable frequency AC voltage. The circuit used in this project consists of four MOSFET's, where each MOSFET is protected by a snubber circuit which does inverting for various frequencies [1], particularly for low frequencies [5]. MOSFET is a three terminal device consisting of Source, Drain and Gate which is usually used for switching purposes. The MOSFET's are triggered by giving pulses at their gate-source terminal. The protection circuit consists of resistor and capacitor, sometimes a diode may also be present (across resistor) which depends on the requirement. This protection circuit preferably protects equipment's against $\mathrm{dv} / \mathrm{dt}$ and over voltages.

Microcontroller: The controlling of inverter is done by using AT89S52 Microcontroller which is an 8-bit microcontroller with 8K Bytes of In-System Programmable (ISP) Flash Memory. The microcontroller IC Atmel's AT89S52 is used to perform the following functions: 
- To provide sinusoidal pulse width modulated pulses using Look-Up-Table (LUT).

- $\quad$ To vary the frequency using timers, with the help of frequency control switches.

- It is also used to display the frequency in the JHD162A [16X2] LCD.

In Fig. 2.2 a single phase, $230 \mathrm{~V}, 50 \mathrm{~Hz}$ Fixed Voltage Fixed Frequency AC source is fed to auto-transformer. The output of the auto-transformer is fed to a full wave bridge rectifier. The output of the rectifier is fed to a RC filter circuit in order to obtain smoothened DC. The smoothened DC which is the output of the filter circuit is then fed to an inverter circuit which consists of 4 MOSFETS in H-bridge arrangement. The MOSFETS are controlled by a microcontroller, to get the desired pulse width modulation and therefore the desired frequency.

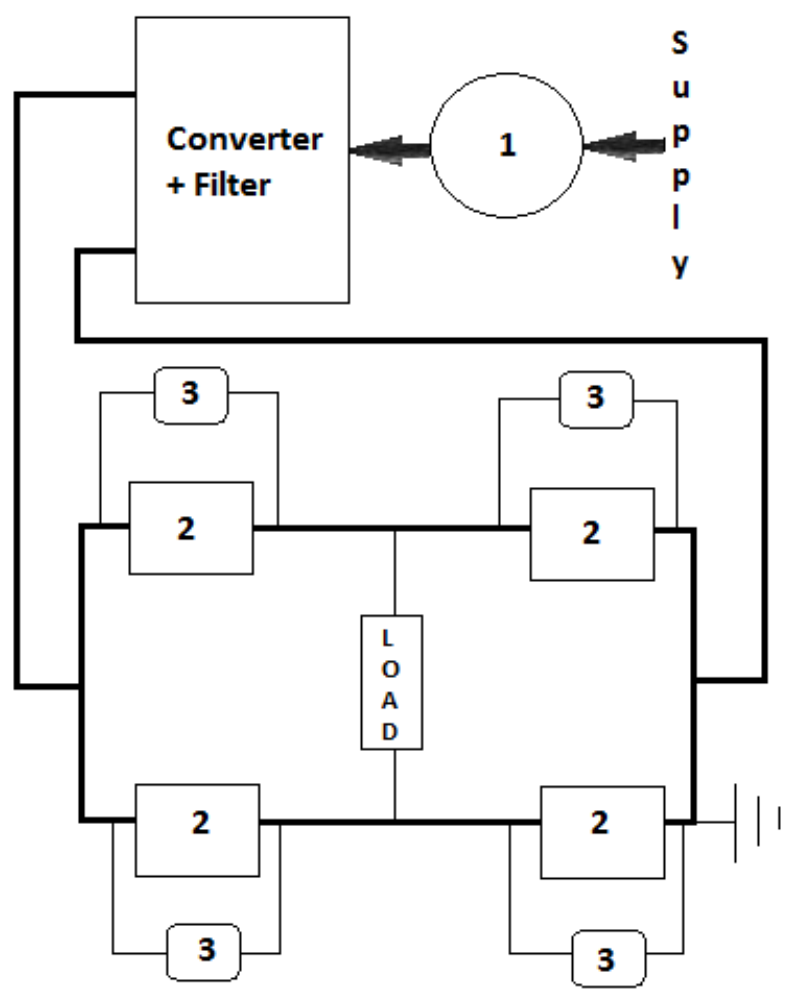

1. Auto-Transformer

2. MOSFET

3. Protective circuit

Fig -2.2 Circuit diagram of H-Bridge Inverter

\subsection{Hardware Design}

Rectifier circuit: The variable voltage output from the Autotransformer is fed as the input to the full wave bridge rectifier to get pulsating $\mathrm{DC}$ as the output. The bridge rectifier IC used is BR-1010. This IC has the capacity to withstand RMS voltage upto $1000 \mathrm{~V}$ and can carry current up to 10 Amps.

Filter circuit: A passive filter (preferably RC filter) is designed to eliminate the ripples present in the output of rectifier. An active filter cannot be used in this case because high voltage filtering is required. By choosing high value of $\mathrm{R}$ and $\mathrm{C}$ the ripple content can be reduced.

Inverter circuit: The output from the filter circuit is fed to the inverter which is in H-bridge arrangement [4]. An $\mathrm{H}$ Bridge or full bridge converter is a switching configuration composed of four switches in an arrangement that resembles an H. By controlling different switches in the bridge, a positive, negative, or zero potential voltage can be placed across a load. When this load is a motor, these states correspond to forward, reverse, and off.

The H-Bridge circuit consists of four switches corresponding to high side left, high side right, low side left, and low side right. There are four possible switch positions that can be used to obtain voltages across the load.

Driver circuit: In order to drive the MOSFETs in H-Bridge arrangement, a MOSFET driver L293D IC is used specifically designed for driving the full-bridge. The L293D is designed to provide bidirectional drive currents of up to $600-\mathrm{mA}$ at voltages from $4.5 \mathrm{~V}$ to $36 \mathrm{~V}$.

Microcontroller Circuit: Microcontroller is used for varying the frequency of the inverter and displaying frequency in LCD. The Microcontroller IC used in the system is ATMEL's AT89S52.The AT89S52 is a low-power, highperformance CMOS 8-bit microcontroller.

LCD Display: The LCD used is a JHD162A, 16 character $\mathrm{x}$ 2 line reflective type Liquid Crystal character display module.

\subsection{Software Design}

Port configuration: PORT 0 - Each pin of this port is connected to a pull-up resistor. This is due to the fact that P0 is an open drain unlike $\mathrm{P} 1, \mathrm{P} 2$ and $\mathrm{P} 3$. This port is configured as an output port and is used to interface LCD with the microcontroller. PORT 1- This port is configured as an output port. The pulse width modulated signals are fed to the L293 driver IC.

Programs encoded in the microcontroller [3] have three sections:

- Interfacing of frequency control switches: Two switches are interfaced to the microcontroller [6] both the switches control the frequency (duration) of pulse width modulated signals by controlling the timer 0 of the microcontroller. One end of the switch which is used to increase the frequency is connected to pin P0.6 of the microcontroller and the other end is connected to $\mathrm{Vcc}(5 \mathrm{~V})$ and the other switch which is used to decrease the frequency is connected in a similar way as the increment switch to pin P0.7 of the microcontroller. So whenever frequency control switch is pressed the pins P0.6 and P0.7 change from their initial logic 0 state to logic 1 state momentarily, the microcontroller senses this change in the logic state 
of the switch and intern the frequency control is achieved.

- $\quad$ Programming of LUT and Interfacing of the Driver [7]: A Look-up table is a table which is predefined in the memory and the program gets some necessary data from the table during runtime. Hence a Look-up table is a table of constants stored in program memory. This type of Look-up table is very important to perform some of the trigonometric operation. Thus, Look-up tables can be used to speed up arithmetic operations. During runtime of the microcontroller, when LUT is not used it makes the calculations complicated, making the program difficult and lengthy. But if the values in memory are stored previously, then one can extract them easily during runtime, which is very easy. The Look-up table may be accessed using the DPTR or PC as a pointer to the start of the table. The A register is used as an index to the table. A look up table which has the value of the time period for which the pulse width modulated signals to be provided is encoded in the ROM area of the microcontroller. So the microcontroller provides a series of pulses to the MOSFETs through the driver.

- Interfacing of LCD to the microcontroller: The LCD module used in this system is JHD162A [8].It is a 16 pin IC with $16 \mathrm{X} 2$ display. The LCD is used to display the frequency of $\mathrm{AC}$ voltage at the output of the system. Pins 11-14 of LCD are used for data transfer. Thus a nibble at a time is transferred to the LCD. Pins 4-5 of LCD are connected to $\mathrm{P} 0.1$ and $\mathrm{P} 0.2$ of microcontroller respectively, these are used to control register select and the enable pin of the LCD. The R/W pin of the LCD is always grounded because the microcontroller will be only writing the data to the LCD.

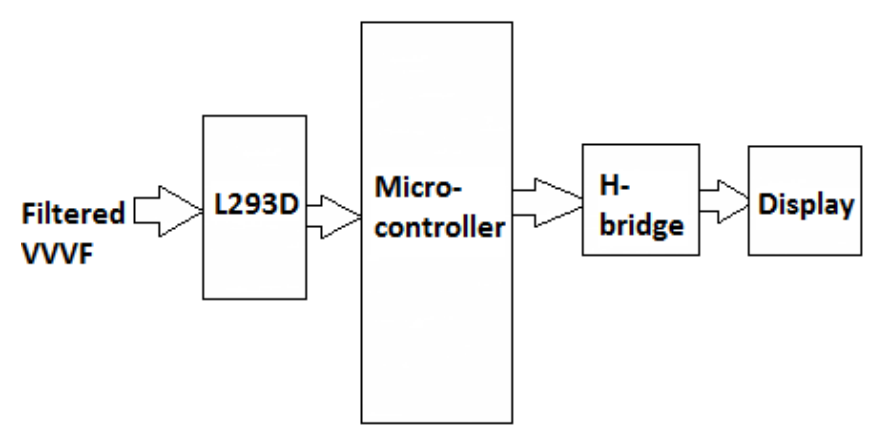

Fig -2.3: Block diagram of Microcontroller circuit

\section{RESULTS}

A variable frequency power source has been developed that can be controlled by pulse width modulation technique. Pulses to control the frequency of power source are generated by ATMEL microcontroller.
Rectifier circuit [4] waveforms: The input to the rectifier IC is fed from the autotransformer. The rectified output (pulsating DC) is fed to the filter circuit in order to remove the $\mathrm{AC}$ component in the rectified wave.

Pulses obtained from AT89S52: Pulse Width Modulated signals obtained from the microcontroller is as shown in the Fig.3.1. This signal is provided to a single MOSFET, similarly 3 such signals are provided to the other 3 MOSFETs. The program is encoded in such a way that, 2 MOSFETs on the same side of the H-bridge will not be receiving the signal simultaneously.

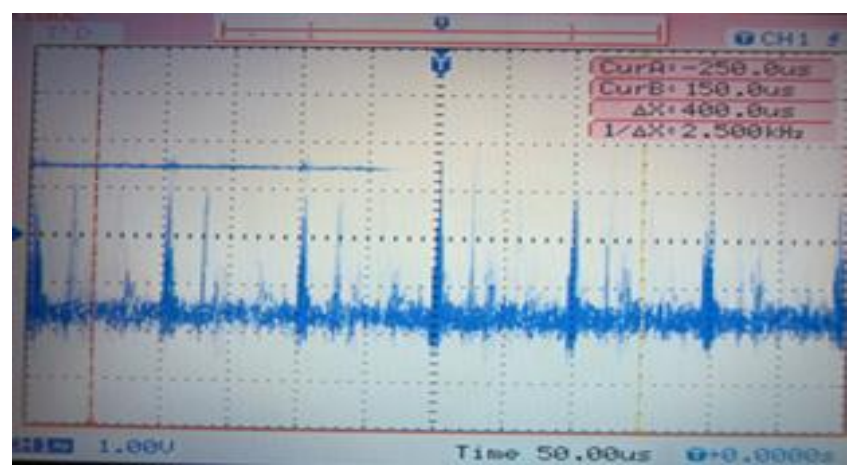

Fig -3.1: Pulse produced from the microcontroller

Pulse produced from the driver circuit: A $5 \mathrm{~V}$ input signal is given to the driver circuit through the microcontroller which is amplified by the driver circuit. The output of the driver circuit used in the proposed model.

Variable frequency waveforms: The final output waveform obtained from the inverter circuit is shown in Fig. 3.2 and the results are summarized in the table 3.1. The frequency generated is displayed in the LCD.

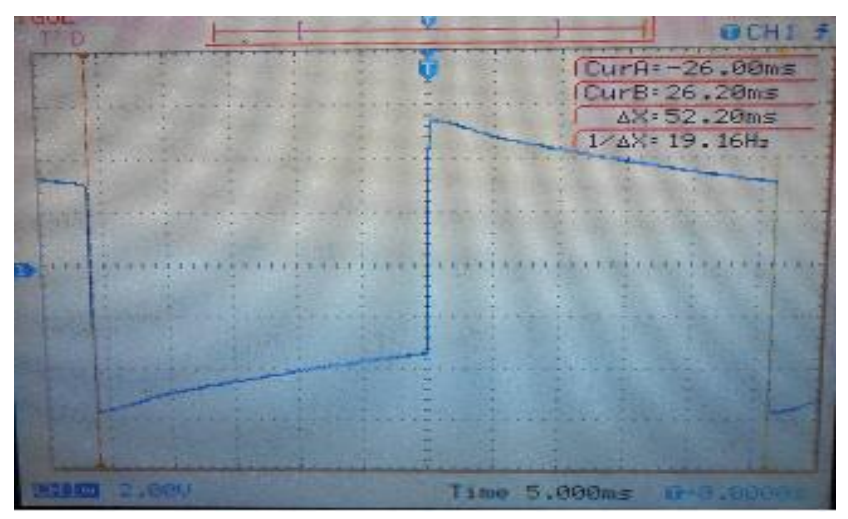

Fig -3.2 Inverter output at $19.16 \mathrm{~Hz}$

Table-3.1

\begin{tabular}{|l|l|}
\hline Frequency & $19.16 \mathrm{~Hz}$ \\
\hline Duration of PWM signal & $52.19 \mathrm{~ms}$ \\
\hline $\begin{array}{l}\text { Frequency Control Switch } \\
\text { Status }\end{array}$ & $\begin{array}{l}\text { Frequency Decrement } \\
\text { Switch is pressed 4 times }\end{array}$ \\
\hline
\end{tabular}




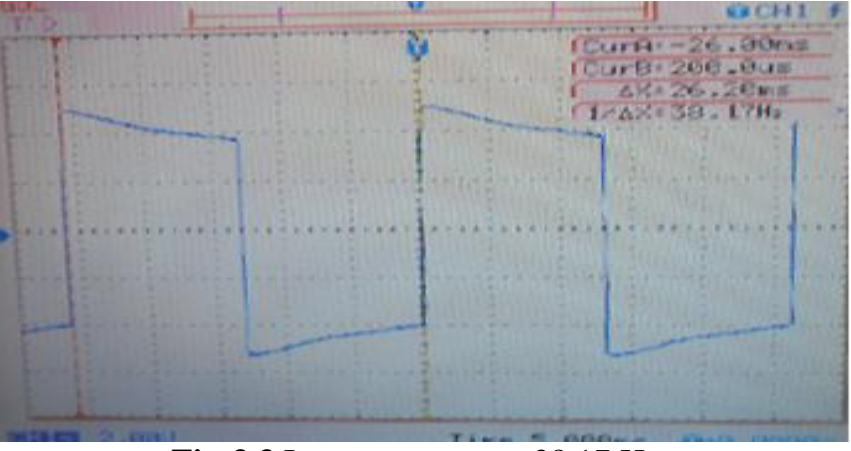

Fig-3.3 Inverter output at $38.17 \mathrm{~Hz}$

Table -3.2

\begin{tabular}{|l|l|}
\hline Frequency & $38.17 \mathrm{~Hz}$ \\
\hline Duration of PWM signal & $26.19 \mathrm{~ms}$ \\
\hline $\begin{array}{l}\text { Frequency Control } \\
\text { Switch Status }\end{array}$ & $\begin{array}{l}\text { Frequency Decrement Switch } \\
\text { is Pressed 3 Times }\end{array}$ \\
\hline
\end{tabular}

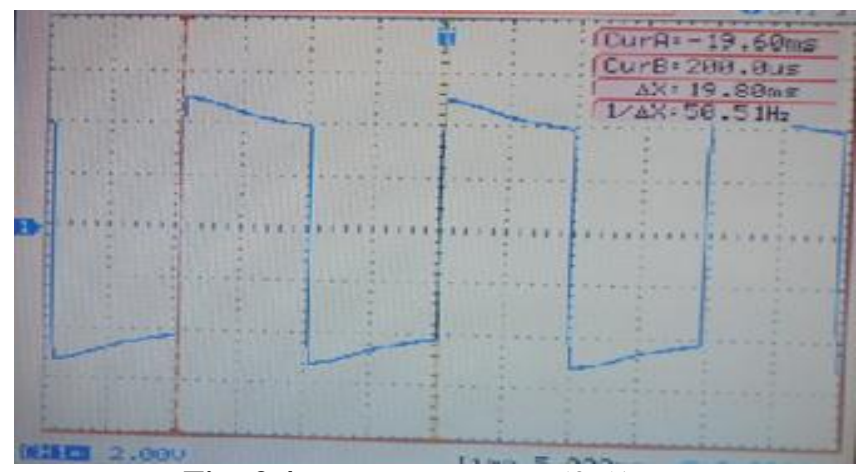

Fig -3.4 Inverter output at $50.51 \mathrm{~Hz}$

Table- 3.3

\begin{tabular}{|l|l|}
\hline Frequency & $50.51 \mathrm{~Hz}$ \\
\hline Duration of PWM signal & $19.79 \mathrm{~ms}$ \\
\hline $\begin{array}{l}\text { Frequency Control Switch } \\
\text { Status }\end{array}$ & $\begin{array}{l}\text { Frequency Decrement } \\
\text { Switch Is Pressed 2 } \\
\text { Times }\end{array}$ \\
\hline
\end{tabular}

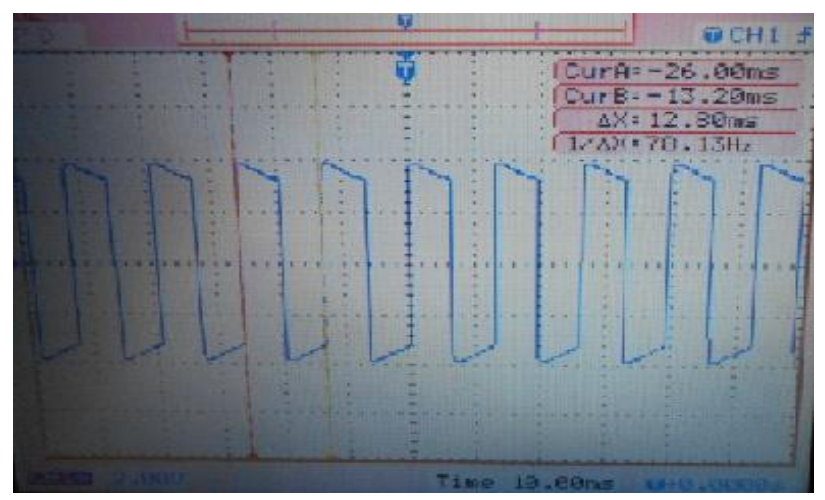

Fig -3.5 Inverter output at $78.31 \mathrm{~Hz}$

Table-3.4

\begin{tabular}{|l|l|}
\hline Frequency & $78.31 \mathrm{~Hz}$ \\
\hline Duration of PWM signal & $12.76 \mathrm{~ms}$ \\
\hline Frequency Control & Frequency Decrement Switch \\
Switch Status & Is Pressed 1 Times \\
\hline
\end{tabular}

\section{CONCLUSIONS}

The design consideration is to obtain a perfect square wave, but acceptable compromise is made with respect to the design parameters of Inverter circuit components, which has led us to a square wave with drop as shown in the figures of section 3. This drop is indicated as slope at both top \& bottom of the wave. The pulse output obtained from the driver circuit assures proper working of the driver circuit. The control over wave frequency and display of these frequency values on the LCD, verifies the programming part of the Microcontroller.

The square wave for different frequencies i.e. for $19 \mathrm{~Hz}, 38$ $\mathrm{Hz}, 50 \mathrm{~Hz} \& 78 \mathrm{~Hz}$ is obtained in the current work, with slight approximations. Thus, one can conclude that further work in this field can be carried out by including appropriate filter circuits, which converts the square wave to sine wave that can be used for various useful purposes like speed control of motors, traction control, iron loss measurement of magnetic specimens and separation of iron losses into hysteresis and eddy current losses etc.

\section{REFERENCES}

[1] K.A. Madi-Ali, M.E. Salem Abozaed, "Microcontroller based Variable Frequency Power Inverter", in: Proc. International Multi Conference of Engineers and Computer Scientists (IMECS), Hong Kong, March 17-19, 2010.

[2] K.S. Low, "A DSP based Variable AC Power Source", IEEE Transaction on Instrumentation and Measurement, 47 (4) (1998), pp. 992-996.

[3] M.A. Mazidi and J.G. Mazidi, "The Programming of 8051 Microcontroller and Embedded Systems using Assembly and C", Pearson Education (Prentice Hall), 2nd edition, 2008.

[4] M.D. Singh and K.B. Khanchandani, "Power Electronics", Tata Mc-Graw Hill Publications, Second edition, 2005.

[5] A text book on "Three Phase Three Level Voltage Source Inverter with Low Switching Frequency based on The Two-Level Inverter Topology" by E. Mahrous, N.A. Rahim, W.P. Hew [5], IET Electrical Power Applications, 1 (4) (2007), pp. 637-641. This helped us in the study of voltage source inverter with High switching frequency.

[6] http://www.wvshare.com/datasheet_html/AT89S52 -PDF.html

[7] www.ti.com/lit/ds/symlink/l293d.pdf

[8] www.egochina.net.cn/eBay/Download/JHD162A.p df

\section{BIOGRAPHIES}

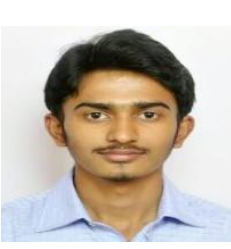

Bangalore, India
Ananya Devraj received his B.E degree in Electrical and Electronics Engineering from Malnad College of engineering, Hassan, Karnataka in 2013 and currently pursing M.Tech in VLSI Design \& Embedded Systems from $\mathrm{T}$ John, 
Darshan $\mathrm{R}$ received his B.E degree in Electrical and Electronics Engineering from Malnad College of engineering, Hassan, Karnataka in 2013 and currently pursing M.Tech in Power System India Engineering from SDMCET, Dharwad, 